\title{
Structure and automorphism groups of Hadamard designs*
}

\author{
Eric Merchant
}

Received: 22 June 2005 / Accepted: 13 January 2006 / Published online: 11 July 2006

(C) Springer Science + Business Media, LLC 2006

\begin{abstract}
Let $n$ be the order of a Hadamard design, and $G$ any finite group. Then there exists many non-isomorphic Hadamard designs of order $2^{12|G|+13} n$ with automorphism group isomorphic to $G$.
\end{abstract}

Keywords Hadamard designs · Automorphisms of designs

\section{Introduction}

One of the fundamental questions concerning any class of combinatorial objects is: given any finite group $G$, can one find a member of the class whose full automorphism group is isomorphic to $G$ ? A survey of such results can be found in [1]. More recently, [7] showed that, for any finite group $G$, and any $q \geq 3$, for all sufficiently large $d$ there exists a symmetric design with the parameters of $\mathcal{P} \mathcal{G}(d, q)$ and with full automorphism group isomorphic to $G$. This paper establishes a stronger version of this when $q=2$ :

Theorem 1.1. Given a finite group $G$ and the existence of a Hadamard design of order $n$, then for all $N>4|G|+2$, there exist at least $\frac{(16 n-2) !}{2^{10} n^{3}}$ non-isomorphic Hadamard designs $\mathcal{D}$ of order $2^{3 N+4} n$ with $\operatorname{Aut}(\mathcal{D}) \cong G$.

The paper is structured as follows: the first 2 sections give basic definitions and notation, as well as structural properties of a "doubling" construction for Hadamard designs. Much of this was covered in [12], where complete proofs can be found.

Section 4 deals with designs obtained from tensor products. It is well known that the tensor (or Kronecker) product of two Hadamard matrices is a Hadamard matrix.

*This research was supported in part by the National Science Foundation.

E. Merchant $(\square)$

On Time Systems Inc., 1850 Millrace Dr., Suite 1, Eugene OR, 97403

e-mail: eric@otsys.com 
Properties of the corresponding product of designs appear not to have been studied. The section concludes with Theorem 4.14, essentially a strong "Krull-Schmidt" theorem which shows that, under suitable conditions, a "tensor factorization" must be unique. This uniqueness provides explicit information about the automorphism group of such a design in terms of the "factor" sub-designs.

Central to the proof of Theorem 1.1 is the construction of rigid Hadamard designs, that is, designs with no non-trivial automorphisms. An infinite family of such designs was previously constructed in [13]. Those designs are of order $2 n$, where $4 n-1$ is a prime power, and are constructed by doubling the Paley designs of [15]. Crucial to that construction is the fact that the full automorphism groups of the Paley designs was given in [6]. Section 5 shows that given any $n$ that is the order of a Hadamard design, there exists a rigid Hadamard design of order $8 n$.

Section 6 surveys known results on GMW designs. These designs have particularly nice automorphism groups, as well as useful structural properties. This combination allows us to construct Hadamard designs with classical parameters having full automorphism group isomorphic to any specified group in Section 7, which concludes with a proof of Theorem 1.1.

\section{Notation and definitions}

This material is discussed at greater length in [12].

Let $\mathcal{D}=(\mathcal{P}, \mathcal{B}, \in)$ be a $2-(4 n-1,2 n-1, n-1)$ Hadamard design, so $v=$ $|\mathcal{P}|=|\mathcal{B}|=b=4 n-1, k=|B|=2 n-1$ for any $B \in \mathcal{B}$, and for $B_{1} \neq B_{2} \in$ $\mathcal{B},\left|B_{1} \cap B_{2}\right|=\lambda=n-1$. Since $\mathcal{D}$ is symmetric, the dual design $\mathcal{D}^{*}=(\mathcal{B}, \mathcal{P}, \ni)$ is a design with the same parameters. The affine completion $\mathcal{D}^{+}$of $\mathcal{D}$ is the following Hadamard 3-design, with parameters $3-(4 n, 2 n, n-1)$ :

- Point-set: $\mathcal{P} \cup\left\{p_{\infty}\right\}$.

- Blocks: $B \cup\left\{p_{\infty}\right\}$ and $B^{c}=\mathcal{P}-B$ for all $B \in \mathcal{B}$.

Note that $\operatorname{Aut}\left(\mathcal{D}^{+}\right)_{p_{\infty}}=\left\{g \in \operatorname{Aut}\left(\mathcal{D}^{+}\right) \mid p_{\infty}^{g}=p_{\infty}\right\} \cong \operatorname{Aut}(\mathcal{D})$. Any point $p$ of the 3 design $\mathcal{D}^{+}$can be used to derive a Hadamard 2-design $\mathcal{D}_{p}^{+}$on the remaining points, and block set the blocks on the $p$. Clearly, $\mathcal{D}_{p_{\infty}}^{+}=\mathcal{D}$, while different points $p$ may induce non-isomorphic 2-designs $\mathcal{D}_{p}$.

Given a block $B \in \mathcal{B}$, the induced design $\mathcal{D}_{(B)}$ on $B$ has as points the points of $B$, and blocks are the point-sets $C \cap B$ for any $C \neq B$. Similarly, given a block $B \in \mathcal{B}$ the residual design $\mathcal{D}^{B}$ off $B$ consists of the points off $B$, and blocks are the sets $C \cap B^{c}$.

Distinct blocks $A, B, C$ of $\mathcal{D}$ form a coline of size 3 if $B \cap C \subset A$. We will denote by $B * C$ the third block on the coline with $B$ and $C$, if such a block exists. Dually, 3 distinct points $p, q, r$ form a line of a Hadamard design if all blocks on both $p$ and $q$ are also on $r=p * q$.

A block $B$ is good if, for any other block $C, B * C$ exists. Equivalently, $B$ is good if and only if $\mathcal{D}_{(B)}$ is a Hadamard design, which is the case if and only if $\mathcal{D}^{B}$ is an affine Hadamard 3-design (for the elementary proof, see [2, Theorem XII. 5.3]). Dually, we say $p$ is a good point if $p * q$ exists for all other points $q$.

Kimberley [10] provides a careful study of good blocks of Hadamard 3-designs. Just as with symmetric designs, a block $B$ of such a design is good if and only if, for 
any block $C \notin\left\{B, B^{c}\right\},(B \triangle C)^{c}$ is also a block. ${ }^{1}$ In that case $\left(B^{c} \triangle C\right)^{c}=B \triangle C$ is also a block, and therefore $B^{c}$ is a good block as well. Thus, the good blocks occur in parallel pairs, and we say $\left\{B, B^{c}\right\}$ is a good parallel class. Given a Hadamard design $\mathcal{D}$, the good blocks of the 3-design $\mathcal{D}^{+}$are the blocks $B \cup\left\{p_{\infty}\right\}$ and $B^{c}$, where $B$ is a good block of $\mathcal{D}$. Conversely, for every good parallel class $\left\{B, B^{c}\right\}$ of an affine Hadamard design $\mathcal{A}$, the member of the class containing $p$ will induce a good block of $\mathcal{A}_{p}$. For, if we have $B$ the member of a good parallel class containing $p$, then given any other block $C$ which contains $p,(B \triangle C)^{c}$ is another block which contains $p$, hence another block of $\mathcal{A}_{p}$. Therefore, although different points $p$ and $q$ may induce non-isomorphic designs $\mathcal{A}_{p}$ and $\mathcal{A}_{q}$, the number of good blocks of $\mathcal{A}_{p}$ and of $\mathcal{A}_{q}$ will equal the number of good parallel classes of $\mathcal{A}$.

A translation of an affine Hadamard design $\mathcal{A}$ is a non-trivial automorphism $f$ with the property that $B^{f}=B$ or $B^{c}$ for all blocks $B$. Clearly, such an automorphism is fixed-point-free (since for some $B, B^{f}=B^{c}$ ) and of order 2 (as $B^{f^{2}}=B$ for all blocks). These were characterized in [12, Lemma 4.4]:

Fact 2.1. Let $p$ and $q$ be distinct points of an affine Hadamard design $\mathcal{A}$. There exists a translation $f \in \operatorname{Aut}(\mathcal{A})$ with $p^{f}=q$ if and only if $q$ is a good point of $\mathcal{A}_{p}$.

An incident point/block pair $p \in B$ is called a flag; if $p$ and $B$ are both good, we will call this a good flag. A non-incident good point/block pair will be called a good anti-flag.

\section{Doubling Hadamard designs}

In this section we define the "doubling" construction first described in [18], and subsequently used by many authors (cf., e.g. [4, 7, 10, 11, 13]). We will then focus on the lines and colines that arise with this construction.

Let $\mathcal{D}_{1}=\left(\mathcal{P}_{1}, \mathcal{B}_{1}\right)$ be a $2-(4 n-1,2 n-1, n-1)$ Hadamard design, and $\mathcal{A}_{2}=$ $\left(\mathcal{P}_{2}, \mathcal{B}_{2}\right)$ a Hadamard $3-(4 n, 2 n, n-1)$ design. Then $\mathcal{A}_{2}$ has $4 n-1=\left|\mathcal{B}_{1}\right|$ parallel classes of blocks. Let $\sigma$ be any bijection from the parallel classes of $\mathcal{A}_{2}$ to $\mathcal{B}_{1}$. Denote by $\overline{B_{2}}$ the parallel class containing $B_{2}$. Then we define the $2-(8 n-1,4 n-1,2 n-1)$ Hadamard design $\mathcal{D}_{1} \sigma \mathcal{A}_{2}$ as follows:

Point-set: $\mathcal{P}_{1} \cup \mathcal{P}_{2}$

Blocks: $B_{\infty}=\mathcal{P}_{1}$ and ${\overline{B_{2}}}^{\sigma} \cup B_{2}$ for all $B \in \mathcal{B}_{2}$.

We first characterize the good blocks that can arise from this construction. A block $B$ of a Hadamard 2-design is good if and only if, for any other block $C,(B \triangle C)^{c}$ is also a block. Therefore $B_{\infty}$ is a good block of $\mathcal{D}_{1} \sigma \mathcal{A}_{2}$, as $\left(\left({\overline{B_{2}}}^{\sigma} \cup B_{2}\right) \triangle B_{\infty}\right)^{c}={\overline{B_{2}}}^{\sigma} \cup B_{2}^{c}$ is a block (clearly $\overline{B_{2}}=\overline{B_{2}^{c}}$ ). It is then easy to see that

$$
\left(\mathcal{D}_{1} \sigma \mathcal{D}_{2}\right)_{\left(B_{\infty}\right)} \cong \mathcal{D}_{1}
$$

\footnotetext{
${ }^{1}$ Throughout, $\triangle$ denotes the symmetric difference of sets, and $X^{c}$ denotes the complement of $X$.
} 
and

$$
\left(\mathcal{D}_{1} \sigma \mathcal{D}_{2}\right)^{B_{\infty}} \cong \mathcal{A}_{2}
$$

The existence of other good blocks of $\mathcal{D}_{1} \sigma \mathcal{A}_{2}$ is a function both of the constituent designs themselves, as well as $\sigma$. Given two blocks of the form ${\overline{B_{2}}}^{\sigma} \cup B_{2}$ and ${\overline{C_{2}}}^{\sigma} \cup C_{2}$, with $C_{2} \notin\left\{B_{2}, B_{2}^{c}\right\}$, we have

$$
\left(\left({\overline{B_{2}}}^{\sigma} \cup B_{2}\right) \triangle\left({\overline{C_{2}}}^{\sigma} \cup C_{2}\right)\right)^{c}=\left(\mathcal{P}_{1}-\left({\overline{B_{2}}}^{\sigma} \triangle{\overline{C_{2}}}^{\sigma}\right)\right) \cup\left(\mathcal{P}_{2}-\left(B_{2} \triangle C_{2}\right)\right) .
$$

Thus, ${\overline{B_{2}}}^{\sigma} \cup B_{2}$ is good if and only if the following 2 conditions are met:

1. Both $B_{2}$ and ${\overline{B_{2}}}^{\sigma}$ are good, so that the sets $\mathcal{P}_{1}-\left({\overline{B_{2}}}^{\sigma} \triangle{\overline{C_{2}}}^{\sigma}\right)$ and $\mathcal{P}_{2}-\left(B_{2} \triangle C_{2}\right)$ are blocks of $\mathcal{B}_{1}$ and $\mathcal{B}_{2}$, respectively, for all blocks $C_{2}$.

2. $\left.\overline{\left(\mathcal{P}_{2}-\left(B_{2} \triangle C_{2}\right)\right.}\right)^{\sigma}=\mathcal{P}_{1}-\left({\overline{B_{2}}}^{\sigma} \triangle \bar{C}_{2}{ }^{\sigma}\right)$ for all blocks $C_{2}$, so that in (3.3), the set on the right is indeed a block of $\mathcal{D}_{1} \sigma \mathcal{A}_{2}{ }^{2}$

Given a $\mathcal{D}_{1}$ and $\mathcal{A}_{2}$ of order $n$, a natural question is: how many non-isomorphic designs can be obtained via the doubling procedure? This question was the main focus of [12], which showed the following (Lemma 3.1):

Fact 3.4. Let $\mathcal{D}_{1} \sigma \mathcal{A}_{2}$ and $\mathcal{D}_{1} \tau \mathcal{A}_{2}$ have $B_{\infty}$ as their only good block. Then $\mathcal{D}_{1} \sigma \mathcal{A}_{2} \cong$ $\mathcal{D} \tau \mathcal{A}_{2}$ if and only if $\sigma \in \overline{G_{2}} \tau G_{1}$, where $G_{1}=\operatorname{Aut}\left(\mathcal{D}_{1}\right)$ in its permutation representation on blocks, and $\overline{G_{2}}=A u t\left(\mathcal{A}_{2}\right)$ in its representation on parallel classes.

Note 3.5. One special case of the doubling procedure deserves mention. Since every block $B$ of a Hadamard design D determines a unique parallel class $\left\{B \cup\left\{p_{\infty}\right\}, B^{c}\right\}$, there is a natural mapping from parallel classes of $\mathcal{D}^{+}$to blocks of $\mathcal{D}$, which we denote $\sigma_{0}$, so $\left\{B \cup\left\{p_{\infty}\right\}, B^{c}\right\}^{\sigma_{0}}=B$.

The resulting design $\mathcal{D} \sigma_{0} \mathcal{D}^{+}$is well-known, and can also be constructed by taking the Hadamard matrix associated with $\mathcal{D}$ (the \pm 1 incidence matrix with an additional row and column of all 1s) and using its Kronecker product with the matrix $\left(\begin{array}{cc}1 & 1 \\ 1 & -1\end{array}\right)$. See Section 4 for more details of that construction.

The existence of good points in these designs was studied in previous work. We mention the two salient facts [12, Theorem 4.3, 4.5]:

Fact 3.6. Let $\mathcal{E}$ be a Hadamard design containing a good anti-flag $p \notin B$. Then $\mathcal{E} \cong \mathcal{D} \sigma_{0} \mathcal{D}^{+}$, where $\mathcal{E}_{(B)} \cong \mathcal{D}$.

Fact 3.7. Let $p \in B$ be a good flag of a Hadamard design $\mathcal{D}$. Then there exists an elation $^{3}$ of $\mathcal{D}$ inducing a translation of the Hadamard 3-design $\mathcal{D}^{B}$.

\footnotetext{
${ }^{2}$ See [14, Lemma 1] for the corresponding characterization in the affine Hadamard design case.

${ }^{3}$ An elation is an automorphism which, given an incident point/block pair, fixes all points on the block and all blocks on the point. 
Much of this paper is dedicated to understanding properties of designs resulting from the "doubling" procedure. Since any such design has at least one good block $B_{\infty}$, a good point of such a design automatically implies the existence of a good flag or a good anti-flag. For that reason, the above facts are used throughout the paper. Another application was given in [12, Theorem 1.3]:

Theorem 3.8. Given a $2-(4 n-1,2 n-1, n-1)$ Hadamard design with $n>2$, there exists a Hadamard design with the same parameters having no good blocks and at most one good point.

In the case where $B_{\infty}$ is the only good block of $\mathcal{D}_{1} \sigma \mathcal{A}_{2}$, the automorphism group can be nicely described. Let $G_{1}=\operatorname{Aut}\left(\mathcal{D}_{1}\right)$ in its permutation representation on blocks, $G_{2}=\operatorname{Aut}\left(\mathcal{A}_{2}\right)$ in its permutation representation on blocks, and $\overline{G_{2}}=\operatorname{Aut}\left(\mathcal{A}_{2}\right)$ in its permutation representation on parallel classes of blocks.

Lemma 3.9. Let $\mathcal{D}_{1} \sigma \mathcal{A}_{2}$ have $B_{\infty}$ as its only good block, and $g \in \operatorname{Aut}\left(\mathcal{D}_{1} \sigma \mathcal{A}_{2}\right)$. Then $g$ induces a $g_{1} \in G_{1}$ on $B_{\infty}$ and a $g_{2} \in G_{2}$ off $B_{\infty}$ satisfying $g_{1}={\overline{g_{2}}}^{\sigma}$.

Conversely, given $g_{1}$ and $g_{2}$ satisfying $g_{1}=\bar{g}_{2}{ }^{\sigma}$, there exists a $g \in \operatorname{Aut}\left(\mathcal{D}_{1} \sigma \mathcal{A}_{2}\right)$ inducing $g_{1}$ and $g_{2}$ on and off $B_{\infty}$, respectively. ${ }^{4}$

Proof: $\Rightarrow$ Since $B_{\infty}$ is the unique good block, $B_{\infty}^{g}=B_{\infty}$. Therefore, $g$ induces an automorphism $g_{1} \in G_{1}$ acting on $B_{\infty}$, and a $g_{2} \in G_{2}$ acting off $B_{\infty}$. Then, for any other block:

$$
\left({\overline{B_{2}}}^{\sigma} \cup B_{2}\right)^{g}={\overline{B_{1}}}^{\sigma g_{1}} \cup B_{2}^{g_{2}}={\overline{B_{2}}}^{\overline{g_{2}} \sigma} \cup B_{2}
$$

which implies

$$
\sigma g_{1}=\overline{g_{2}} \sigma
$$

$\Leftarrow$ Suppose $g_{1}={\overline{g_{2}}}^{\sigma}$ for $g_{1} \in G_{1}, \overline{g_{2}} \in \overline{G_{2}}$. Fix $g_{2} \in G_{2}$, an arbitrary pre-image of $\overline{g_{2}}$. We then define an automorphism $g$ acting as $g_{1}$ on $B_{\infty}$ and as $g_{2}$ off $B_{\infty}$ as follows:

$$
\left({\overline{B_{2}}}^{\sigma} \cup B_{2}\right)^{g}={\overline{B_{2}}}^{\sigma g_{1}} \cup B_{2}^{g_{2}}={\overline{B_{2}}}^{\sigma \sigma^{-1} \overline{g_{2}} \sigma} \cup B_{2}^{g_{2}}={\overline{B_{2}}}^{\overline{g_{2}} \sigma} \cup B_{2}^{g_{2}} .
$$

Which is a block of $\mathcal{D}_{1} \sigma \mathcal{A}_{2}$, proving that $g$ induces an automorphism.

Corollary 3.10. Let $\mathcal{D}_{1} \sigma \mathcal{A}_{2}$ have $B_{\infty}$ as its only good block. If Aut $\left(\mathcal{A}_{2}\right)$ contains no translations, then:

$$
\operatorname{Aut}\left(\mathcal{D}_{1} \sigma \mathcal{A}_{2}\right) \cong G_{1} \cap{\overline{G_{2}}}^{\sigma} .
$$

\footnotetext{
${ }^{4}$ Similar ideas appear in $[7,13]$.
} 
Proof: Since $G_{2}$ contains no translations, the homomorphism $G_{2} \rightarrow \overline{G_{2}}$ is injective, so every $g_{2}$ induces a unique $\overline{g_{2}}$. Thus, Lemma 3.9 provides the bijection between $\operatorname{Aut}\left(\mathcal{D}_{1} \sigma \mathcal{A}_{2}\right)$ and $G_{1} \cap{\overline{G_{2}}}^{\sigma}$.

Lemma 3.11. Let $p$ be a point of $\mathcal{D}_{1}$ and $q, r$ be points of $\mathcal{A}_{2}$. Then $\{p, q, r\}$ is a line of $\mathcal{D}_{1} \sigma \mathcal{A}_{2}$ if and only if, for the $2 n-1$ blocks $B$ of $\mathcal{A}_{2}$ with $q, r \in B$, we have $p \in \bar{B}^{\sigma}$.

Proof: $\Rightarrow$ If $\{p, q, r\}$ is a line of $\mathcal{D}_{1} \sigma \mathcal{A}_{2}$, then every time $p, q \in \bar{B}^{\sigma} \cup B$, we have $r \in \bar{B}^{\sigma} \cup B$, so $q, r \in B$ and $p \in \bar{B}^{\sigma}$.

$\Leftarrow$ Given such a $\sigma$, if $q, r \in \bar{B}^{\sigma} \cup B$ then $p \in \bar{B}^{\sigma} \cup B$. Thus, $\{p, q, r\}$ form a line of $\mathcal{D}_{1} \sigma \mathcal{A}_{2}$.

Corollary 3.12. Let $\mathcal{D}_{1}$ be a Hadamard design of order $n>2$, and $\mathcal{A}_{2}$ an affine Hadamard design of order $n$. Then there exists a $\sigma$ such that $\mathcal{D}_{1} \sigma \mathcal{A}_{2}$ has at least 1 point on no lines of size 3 .

Proof: By Lemma 3.11, given a point $p$ of $\mathcal{D}_{1}$ and points $q, r$ of $\mathcal{A}_{2}$, there are $(2 n) !(2 n-1)$ ! choices of $\sigma$ for which $\mathcal{D}_{1} \sigma \mathcal{D}_{2}$ has $\{p, q, r\}$ as a line. Fixing $q$, but letting $p$ and $r$ vary, we see that there are $(4 n-1) !-(4 n-1)^{2}(2 n) !(2 n-1) !>0$ choices of $\sigma$ for which $\mathcal{D}_{1} \sigma \mathcal{A}_{2}$ has no lines of size 3 through $q$.

\section{The tensor product}

If $\mathcal{D}_{1}=\left(\mathcal{P}_{1}, \mathcal{B}_{1}\right)$ and $\mathcal{D}_{2}=\left(\mathcal{P}_{2}, \mathcal{B}_{2}\right)$ are Hadamard designs, we denote by $\mathcal{D}_{1} \otimes \mathcal{D}_{2}$ the Hadamard design on $\mathcal{P}_{1} \cup \mathcal{P}_{2} \cup\left(\mathcal{P}_{1} \times \mathcal{P}_{2}\right)$, with blocks of the following form.

$$
\begin{aligned}
B_{1}^{*} & =B_{1} \cup \mathcal{P}_{2} \cup\left(B_{1} \times \mathcal{P}_{2}\right) \\
B_{2}^{*} & =\mathcal{P}_{1} \cup B_{2} \cup\left(\mathcal{P}_{1} \times B_{2}\right) \\
\left(B_{1}, B_{2}\right) & =\left(B_{1}^{*} \triangle B_{2}^{*}\right)^{c} .
\end{aligned}
$$

We will need the line structure of the design (colines are described similarly).

Observation 4.4. The lines of size 3 in the design $\mathcal{D}=\mathcal{D}_{1} \otimes \mathcal{D}_{2}$ are of the following types, where $\left\{x_{i}, y_{i}, z_{i}\right\}$ is a line of $\mathcal{D}_{i}$ of size 3 and $p_{i}$ is an arbitrary point of $\mathcal{D}_{i}$ :

i. $\left\{x_{i}, y_{i}, z_{i}\right\}$

ii. $\left\{\left(x_{1}, x_{2}\right),\left(y_{1}, y_{2}\right),\left(z_{1}, z_{2}\right)\right\}$

iii. $\left\{\left(x_{1}, p_{2}\right),\left(y_{1}, p_{2}\right), z_{1}\right\}$ or $\left\{\left(p_{1}, x_{2}\right),\left(p_{1}, y_{2}\right), z_{2}\right\}$

iv. $\left\{p_{1}, p_{2},\left(p_{1}, p_{2}\right)\right\}$

Two special cases are worth mentioning. First, the trivial case of an "empty" Hadamard design $\mathcal{E}$ with no points or blocks. Clearly, $\mathcal{D} \otimes \mathcal{E}=\mathcal{D}$ (just as taking a Kronecker product with the matrix [1] leaves the original matrix unchanged) and this case shall hereafter be ignored. More interesting is the tensor product with the Springer 
design consisting of one point and block which are non-incident. As mentioned in Section 3 above, this corresponds to the doubling construction of $\mathcal{D} \sigma_{0} \mathcal{D}^{+}$.

Corollary 4.5. If $\mathcal{D} \cong \mathcal{D}_{1} \otimes \mathcal{D}_{2}$, then every point of $\mathcal{D}$ is on at least one line of size 3 .

Proof: Lines of the form $\left\{p_{1}, p_{2},\left(p_{1}, p_{2}\right)\right\}$ cover all points.

For any Hadamard design $\mathcal{D}$ and point $p$, define:

$$
p^{\perp}=\{p\} \cup\{q \mid p * q \text { exists }\},
$$

and for any $X \subseteq \mathcal{P}$ :

$$
X^{\perp}=\bigcap_{p \in X} p^{\perp}
$$

Lemma 4.6. Let $p_{1}$ be a point of $\mathcal{D}_{1} \otimes \mathcal{D}_{2}$, and let $\perp_{1}$ denote the perp structure of $\mathcal{D}_{1}$. Then

$$
p_{1}^{\perp}=p_{1}^{\perp_{1}} \cup \mathcal{P}_{2} \cup\left(p_{1}^{\perp_{1}} \times \mathcal{P}_{2}\right)
$$

Proof: Lines of type $i$ give $p_{1}^{\perp} \cap \mathcal{P}_{1}=p_{1}^{\perp_{1}}$. All points of $\mathcal{P}_{2}$ are contained in $p_{1}^{\perp}$ courtesy of lines of type iii. Finally, $p_{1}^{\perp} \cap\left(\mathcal{P}_{1} \times \mathcal{P}_{2}\right)=p_{1}^{\perp_{1}} \times \mathcal{P}_{2}$ as lines of type $i v$ connect $p_{1}$ and a point of the form $\left(q_{1}, p_{2}\right)$ if and only if $q_{1} \in p_{1}^{\perp_{1}}$.

Of course, we can similarly characterize $p_{2}^{\perp}$.

Lemma 4.7. Let $\left(p_{1}, p_{2}\right)$ be a point of $\mathcal{D}_{1} \otimes \mathcal{D}_{2}$. Then

$$
\left(p_{1}, p_{2}\right)^{\perp}=p_{1}^{\perp} \cap p_{2}^{\perp}=p_{1}^{\perp_{1}} \cup p_{2}^{\perp_{2}} \cup\left(p_{1}^{\perp_{1}} \times p_{2}^{\perp_{2}}\right) .
$$

Proof: $p_{1}^{\perp_{1}} \subseteq\left(p_{1}, p_{2}\right)^{\perp}$ as lines of type $i i i$ will exist whenever the corresponding line exists in $\mathcal{D}_{1}$. Also, $p_{1} \in\left(p_{1}, p_{2}\right)^{\perp}$ via the unique line of type $i v$ through $\left(p_{1}, p_{2}\right)$. Similarly for $p_{2}^{\perp_{2}} \subseteq\left(p_{1}, p_{2}\right)^{\perp}$. Finally, lines of type $i i$ show that $\left(p_{1}, p_{2}\right)^{\perp} \cap\left(\mathcal{P}_{1} \times \mathcal{P}_{2}\right)=p_{1}^{\perp_{1}} \times p_{2}^{\perp_{2}}$.

Lemma 4.8. The good points of $\mathcal{D}_{1} \otimes \mathcal{D}_{2}$ are exactly those of the form $p_{1}, p_{2}$, or $\left(p_{1}, p_{2}\right)$ where $p_{i}$ is a good point of $\mathcal{D}_{i}$.

Proof: Lemma 4.6 shows that $p_{1}$ is good if and only if $p_{1}^{\perp_{1}}=\mathcal{P}_{1}$, i.e., if and only if $p_{1}$ is a good point of $\mathcal{D}_{1}$. Similarly with a point $p_{2}$. For a point of the form $\left(p_{1}, p_{2}\right)$ to be good, Lemma 4.7 shows that both $p_{1}$ and $p_{2}$ will necessarily be good as well. 
Corollary 4.9. Let $\mathcal{D}=\mathcal{D}_{1} \otimes \mathcal{D}_{2}$ where $\mathcal{D}_{2}$ has no good points. If $p$ is a point of $\mathcal{D}$, then $p^{\perp} \supseteq \mathcal{P}_{2}$ if and only if $p \in \mathcal{P}_{1}$.

Lemma 4.10. Let $\mathcal{D}_{1} \otimes \mathcal{D}_{2}$ have a point-transitive automorphism group. Then $\mathcal{D}_{1} \otimes$ $\mathcal{D}_{2}$ is isomorphic to a projective space over $\mathbb{F}_{2}$.

Proof: For any $p_{1} \in \mathcal{P}_{1}$ and $p_{2} \in \mathcal{P}_{2}$, there exists an automorphism $g \in \operatorname{Aut}\left(\mathcal{D}_{1} \otimes\right.$ $\left.\mathcal{D}_{2}\right)$ so $p_{1}^{g}=\left(p_{1}, p_{2}\right)$. Therefore, $\left|p_{1}^{\perp}\right|=\left|\left(p_{1}, p_{2}\right)^{\perp}\right|$, which implies by Lemma 4.7 $p_{1}^{\perp} \subseteq p_{2}^{\perp}$. Thus, $\mathcal{P}_{2} \subseteq p_{2}^{\perp}$, so $p_{2}$ is a good point of $\mathcal{D}_{2}$ and hence of $\mathcal{D}_{1} \otimes \mathcal{D}_{2}$ by Lemma 4.8. Since $\operatorname{Aut}\left(\mathcal{D}_{1} \otimes \mathcal{D}_{2}\right)$ is transitive, all points of $\mathcal{D}_{1} \otimes \mathcal{D}_{2}$ are good. This implies that $\mathcal{D}_{1} \otimes \mathcal{D}_{2}$ is a projective space by the Dembowski-Wagner Theorem [2, Theorem XII.2.10].

Theorem 4.11. Given a Hadamard design $\mathcal{D}$, we have $\mathcal{D} \cong \mathcal{D}_{1} \otimes \mathcal{D}_{2}$ where $\mathcal{D}_{i}=$ $\left(\mathcal{P}_{i}, \mathcal{B}_{i}\right)$ if and only if the point set and the block set of $\mathcal{D}$ can be partitioned as $\mathcal{P}=\mathcal{P}_{1} \cup \mathcal{P}_{2} \cup\left(\mathcal{P}_{1} \times \mathcal{P}_{2}\right)$ and $\mathcal{B}=\mathcal{B}_{1} \cup \mathcal{B}_{2} \cup\left(\mathcal{B}_{1} \times \mathcal{B}_{2}\right)$ satisfying the following:

1. The incidence structures $\left(\mathcal{P}_{i}, \mathcal{B}_{i}\right)$ are both induced 2-designs of $\mathcal{D} .^{5}$

2. For all $p_{1} \in \mathcal{P}_{1}$ and $B_{2} \in \mathcal{B}_{2}$ we have $p_{1} \in B_{2}$, and for all $p_{2} \in \mathcal{P}_{2}$ and $B_{1} \in \mathcal{B}_{1}$ we have $p_{2} \in B_{1}$

3. For every $\left(p_{1}, p_{2}\right) \in \mathcal{P}_{1} \times \mathcal{P}_{2},\left(p_{1}, p_{2}\right)=p_{1} * p_{2}$. Dually, every block $\left(B_{1}, B_{2}\right) \in$ $\mathcal{B}_{1} \times \mathcal{B}_{2},\left(B_{1}, B_{2}\right)=B_{1} * B_{2}$.

Proof: The forward implication is immediate, by the definition of $\mathcal{D}_{1} \otimes \mathcal{D}_{2}$. For the converse, first note that if such sub-designs of $\mathcal{D}$ exist, they must themselves be symmetric designs. The existence of the partitions shows:

$$
v_{1}+v_{2}+v_{1} v_{2}=v=b=b_{1}+b_{2}+b_{1} b_{2} .
$$

Combined with Fisher's Inequality: $v_{i} \leq b_{i}$ for any 2-design gives $v_{i}=b_{i}$, implying that both induced sub-designs are symmetric. To see that both designs are Hadamard, consider any block $B_{1} \in \mathcal{B}_{1}$. Since $\left\{p_{1}, p_{2},\left(p_{1}, p_{2}\right)\right\}$ forms a line by condition 3 , we have $\left(p_{1}, p_{2}\right) \in B_{1} \Leftrightarrow p_{1} \in B_{1}$. Thus, since $\mathcal{D}$ is Hadamard, we have:

$$
\left|B_{1}\right|=k_{1}+v_{2}+k_{1} v_{2}=\frac{1}{2}(v+1)-1=\frac{1}{2}\left(v_{1}+v_{2}+v_{1} v_{2}+1\right)-1 .
$$

Which gives:

$$
k_{1}+1=\frac{1}{2}\left(v_{1}+1\right)
$$

which shows that $\mathcal{D}_{1}$ is Hadamard. Similarly, so is $\mathcal{D}_{2}$.

\footnotetext{
${ }^{5}$ By conventional abuse of notation, $\left(\mathcal{P}_{i}, \mathcal{B}_{i}\right)$ is the design consisting of the point set $\mathcal{P}_{i}$ and blocks being the intersection of $\mathcal{B}_{i}$ with the point set. Note that $\left(\mathcal{P}_{i}, \mathcal{B}_{i}\right)$ may be empty or tirvial, as indicated above. 
Also, condition 2 and the lines in 3 show that the blocks of $\mathcal{B}_{1}$ consist of the same points required by Eq. (4.1). Similarly for Eq. (4.2), and the colines of condition 3 ensure that the blocks of the form $\left(B_{1}, B_{2}\right)$ satisfy Eq. (4.3).

If $\mathcal{D}$ contains subdesigns $\mathcal{D}_{1}$ and $\mathcal{D}_{2}$ satisfying the conditions of Theorem 4.11 , then we say $\mathcal{D}=\mathcal{D}_{1} \otimes \mathcal{D}_{2}$ is an internal tensor product. The associativity of this product follows from the corresponding statement about matrices:

Lemma 4.12. For Hadamard designs $\mathcal{D}_{1}, \mathcal{D}_{2}$ and $\mathcal{D}_{3}$,

$$
\left(\mathcal{D}_{1} \otimes \mathcal{D}_{2}\right) \otimes \mathcal{D}_{3}=\mathcal{D}_{1} \otimes\left(\mathcal{D}_{2} \otimes \mathcal{D}_{3}\right)
$$

Proof: The design on the left has point-set:

$$
\left(\mathcal{P}_{1} \cup \mathcal{P}_{2} \cup\left(\mathcal{P}_{1} \times \mathcal{P}_{2}\right)\right) \cup \mathcal{P}_{3} \cup\left(\left(\mathcal{P}_{1} \cup \mathcal{P}_{2} \cup\left(\mathcal{P}_{1} \times \mathcal{P}_{2}\right)\right) \times \mathcal{P}_{3}\right)
$$

and blocks similarly. The subdesigns $\left(\mathcal{P}_{1}, \mathcal{B}_{1}\right)$ and $\left(\mathcal{P}_{2} \cup \mathcal{P}_{3} \cup\left(\mathcal{P}_{2} \times \mathcal{P}_{3}\right), \mathcal{B}_{2} \cup \mathcal{B}_{3} \cup\right.$ $\left.\left(\mathcal{B}_{2} \times \mathcal{B}_{3}\right)\right)$ satisfy the conditions of Theorem 4.11 .

Theorem 4.13. If $\mathcal{D}=\mathcal{D}_{1} \otimes \mathcal{D}_{2}=\mathcal{D}_{1}^{\prime} \otimes \mathcal{D}_{2}^{\prime}$ and $\mathcal{D}$ contains no good points, then:

$$
\mathcal{D}=\left(\mathcal{D}_{1} \cap \mathcal{D}_{1}^{\prime}\right) \otimes\left(\mathcal{D}_{1} \cap \mathcal{D}_{2}^{\prime}\right) \otimes\left(\mathcal{D}_{2} \cap \mathcal{D}_{1}^{\prime}\right) \otimes\left(\mathcal{D}_{2} \cap \mathcal{D}_{2}^{\prime}\right)
$$

where $\mathcal{D}_{i} \cap \mathcal{D}_{j}^{\prime}$ is the induced subdesign on $\left(\mathcal{P}_{i} \cap \mathcal{P}_{j}^{\prime}, \mathcal{B}_{i} \cap \mathcal{B}_{j}^{\prime}\right){ }^{6}$

Proof: It suffices to show that $\mathcal{D}_{1}=\left(\mathcal{D}_{1} \cap \mathcal{D}_{1}^{\prime}\right) \otimes\left(\mathcal{D}_{1} \cap \mathcal{D}_{2}^{\prime}\right) ; \mathcal{D}_{2}$ will decompose similarly. We have:

$$
\mathcal{P}_{1}=\left(\mathcal{P}_{1} \cap \mathcal{P}_{1}^{\prime}\right) \cup\left(\mathcal{P}_{1} \cap \mathcal{P}_{2}^{\prime}\right) \cup\left(\mathcal{P}_{1} \cap\left(\mathcal{P}_{1}^{\prime} \times \mathcal{P}_{2}^{\prime}\right)\right)
$$

as a partition of $\mathcal{P}_{1}$. We first show that this partition satisfies Property 3 of Theorem 4.11. Let $p_{1}=\left(p_{1}^{\prime}, p_{2}^{\prime}\right)$, so $p_{1}=p_{1}^{\prime} * p_{2}^{\prime}$. We need to show that $p_{1}^{\prime}, p_{2}^{\prime} \in \mathcal{P}_{1}$. By Lemmas 4.6 and 4.7 , we have:

$$
\mathcal{P}_{2} \subseteq p_{1}^{\perp}=\left(p_{1}^{\prime}, p_{2}^{\prime}\right)^{\perp}=p_{1}{ }^{\perp} \cap p_{2}{ }^{\perp}
$$

Thus, $\mathcal{P}_{2} \subseteq p_{1}^{\prime \perp}$, so $p_{1}{ }^{\perp} \in \mathcal{P}_{1}$ by Corollary 4.9. Clearly, the same argument shows that $p_{2}^{\prime} \in \mathcal{P}_{1}$, so we have verified Property 3 for the points of $\mathcal{P}_{1}$. For the dual condition, suppose $B_{1}=\left(B_{1}^{\prime}, B_{2}^{\prime}\right) \in \mathcal{B}_{1}$. We need to show $B_{1}^{\prime *}, B_{2}^{\prime *} \in \mathcal{B}_{1}$. It suffices to show that $\mathcal{P}_{2} \subset B_{1}^{\prime *}$ as the blocks of $\mathcal{B}_{1}$ are the only blocks which contain all points of $\mathcal{P}_{2}$, and we already have $\mathcal{P}_{2} \subset B_{1}^{*}=\left(B_{1}^{\prime}, B_{2}^{\prime}\right)$. Let $q_{2} \in \mathcal{P}_{2}$, and we consider the following 3 cases:

\footnotetext{
${ }^{6}$ Note that the $\mathcal{D}_{i} \cap \mathcal{D}_{j}$ may be trivial, or even empty.
} 
1. $q_{2}=q_{1}^{\prime} \in \mathcal{P}_{2} \cap \mathcal{P}_{1}^{\prime}$. But, $q_{1}^{\prime} \in\left(B_{1}^{\prime}, B_{2}^{\prime}\right)$ implies $q_{1}^{\prime} \in B_{1}{ }^{\prime *}$.

2. $q_{2}=q_{2}^{\prime} \in \mathcal{P}_{2} \cap \mathcal{P}_{2}^{\prime}$. Then $q_{2}^{\prime} \in B_{1}{ }^{* *}$ is immediate.

3. $q_{2}=\left(q_{1}^{\prime}, q_{2}^{\prime}\right) \in \mathcal{P}_{2} \cap\left(\mathcal{P}_{1}^{\prime} \times \mathcal{P}_{2}^{\prime}\right)$. By the argument above, this means $q_{1}^{\prime}, q_{2}^{\prime} \in \mathcal{P}_{2} \subset$ $\left(B_{1}^{\prime}, B_{2}^{\prime}\right)$, so $q_{1}^{\prime} \in B_{1}^{\prime *}$, implying $\left(q_{1}^{\prime}, q_{2}^{\prime}\right) \in B_{1}^{\prime *}$.

The same argument shows $B_{2}^{\prime} \in \mathcal{B}_{1}$, completing the proof of Property 3 .

Property 2 of Theorem 4.11 is immediate, as any point of $\mathcal{P}_{1} \cap \mathcal{P}_{1}^{\prime}$ is contained in every block of $\mathcal{B}_{1} \cap \mathcal{B}_{2}^{\prime}$ simply by the incidence relations in $\mathcal{D}_{1}^{\prime} \otimes \mathcal{D}_{2}^{\prime}$.

For Property 1 of Theorem 4.11, first note $\mathcal{D}_{1}$ is a 2-design. Any two points $p, q \in$ $\mathcal{P}_{1} \cap \mathcal{P}_{1}^{\prime}$ lie on $\lambda_{1}$ blocks of $\mathcal{B}_{1}$. But, $\mathcal{B}_{1}=\left(\mathcal{B}_{1} \cap \mathcal{B}_{1}^{\prime}\right) \cup\left(\mathcal{B}_{1} \cap \mathcal{B}_{2}^{\prime}\right) \cup\left(\mathcal{B}_{1} \cap\left(\mathcal{B}_{1}^{\prime} \times \mathcal{B}_{2}^{\prime}\right)\right)$. Suppose $p$ and $q$ lie on $\lambda_{p q}$ blocks of $\mathcal{B}_{1} \cap \mathcal{B}_{1}^{\prime}$. Then they lie on every block of $\mathcal{B}_{1} \cap \mathcal{B}_{2}^{\prime}$ by Property 2 (say this set has size $\beta$ ), and Property 3 gives us that the blocks of $\mathcal{B}_{1} \cap\left(\mathcal{B}_{1}^{\prime} \times \mathcal{B}_{2}^{\prime}\right)$ are on unique colines with a block from each of the first two sets, so $p$ and $q$ must lie on $\lambda_{p q} \beta$ blocks of that type. Therefore:

$$
\lambda_{1}=\lambda_{p q}+\beta+\lambda_{p q} \beta
$$

Since $\lambda_{1}$ and $\beta$ are both independent of the choice of $p$ and $q, \lambda_{p q}$ must be too. In other words, $\left(\mathcal{P}_{1} \cap \mathcal{P}_{1}^{\prime}, \mathcal{B}_{1} \cap \mathcal{B}_{1}^{\prime}\right)$ is an induced 2-design of $\mathcal{D}_{1}$. The same argument gives $\left(\mathcal{P}_{1} \cap \mathcal{P}_{2}^{\prime}, \mathcal{B}_{1} \cap \mathcal{B}_{2}^{\prime}\right)$ a 2-design, completing the proof.

In the above theorem, "no good points" can be replaced with the hypothesis "no good blocks" by duality.

If $\mathcal{D} \neq \mathcal{D}_{1} \otimes \mathcal{D}_{2}$ for any non-empty $\mathcal{D}_{1}, \mathcal{D}_{2}$, we say $\mathcal{D}$ is tensor-indecomposable. The following corollary demonstrates that, in the absence of good points, a design decomposes into unique tensor factors. This is in contrast to the classical case of $\mathcal{P G}(d, 2)$, which can be decomposed into complementary subspaces in many ways.

Corollary 4.14. Let

$$
\mathcal{D}=\bigotimes_{i=1}^{n} \mathcal{D}_{i}=\bigotimes_{i=1}^{m} \mathcal{D}_{i}^{\prime}
$$

where the $\mathcal{D}_{i}$ and $\mathcal{D}_{i}^{\prime}$ are tensor-indecomposable, and $\mathcal{D}$ has no good points.

Then there exists a bijection $f:\{1, \ldots, n\} \rightarrow\{1, \ldots, m\}$ satisfying $\mathcal{D}_{i^{f}} \cong \mathcal{D}_{i}^{\prime}$.

Proof: We have:

$$
\mathcal{D}=\mathcal{D}_{1} \otimes \bigotimes_{i=2}^{n} \mathcal{D}_{i}=\mathcal{D}_{1}^{\prime} \otimes \bigotimes_{i=2}^{m} \mathcal{D}_{i}^{\prime}
$$

If $\mathcal{D}_{1}=\mathcal{D}_{1}^{\prime}$, we are done by induction. Otherwise, Theorem 4.13 gives:

$$
\mathcal{D}_{1}=\left(\mathcal{D}_{1} \cap \mathcal{D}_{1}^{\prime}\right) \otimes\left(\mathcal{D}_{1} \cap \bigotimes_{i=2}^{m} \mathcal{D}_{i}^{\prime}\right)
$$


Since $\mathcal{D}_{1}$ is tensor-indecomposable, we must have $\mathcal{D}_{1}=\mathcal{D}_{1} \cap \otimes_{i=2}^{m} \mathcal{D}_{i}^{\prime}$. Another application of Theorem 4.13 yields:

$$
\bigotimes_{i=2}^{m} \mathcal{D}_{i}^{\prime}=\left(\mathcal{D}_{1} \cap \bigotimes_{i=2}^{m} \mathcal{D}_{i}^{\prime}\right) \otimes\left(\bigotimes_{i=2}^{n} \mathcal{D}_{i} \cap \bigotimes_{i=2}^{m} \mathcal{D}_{i}^{\prime}\right)=\mathcal{D}_{1} \otimes\left(\bigotimes_{i=2}^{n} \mathcal{D}_{i} \cap \bigotimes_{i=2}^{m} \mathcal{D}_{i}^{\prime}\right)
$$

By induction, this decomposition is unique, so $m-1=n-1$, and for some $i$, we have $\mathcal{D}_{i}^{\prime}=\mathcal{D}_{1}$.

Similarly, determining a factorization determines the automorphism group in terms of the automorphism groups of the factors involved.

Corollary 4.15. Let $\mathcal{D}$ have no good points and

$$
\mathcal{D}=\bigotimes_{i=1}^{n_{1}} \mathcal{D}_{1} \otimes \bigotimes_{i=1}^{n_{2}} \mathcal{D}_{2} \otimes \cdots \otimes \bigotimes_{i=1}^{n_{m}} \mathcal{D}_{m}
$$

for tensor-indecomposable designs $\mathcal{D}_{i}$. Then:

$$
\operatorname{Aut}(\mathcal{D}) \cong \operatorname{Aut}\left(\mathcal{D}_{1}\right)<S_{n_{1}} \times \operatorname{Aut}\left(\mathcal{D}_{2}\right)<S_{n_{2}} \times \cdots \times \operatorname{Aut}\left(\mathcal{D}_{m}\right)<S_{n_{m}}
$$

Proof: Any automorphism of $\mathcal{D}$ maps a decomposition to a decomposition, but Theorem 4.14 ensures that this decomposition is unique. Thus, the above group is the only possibility.

The corollary of the following Lemma gives a necessary condition for designs of the form $\mathcal{D}_{1} \sigma \mathcal{A}_{2}$ to be tensor-decomposable. Recall the definition of a block $B^{*}$ from Eq. (4.1).

Lemma 4.16. Let $\mathcal{D}=\mathcal{D}_{1} \otimes \mathcal{D}_{2}$ and let $B$ be a good block of $\mathcal{D}_{1}$. Then $\mathcal{D}_{\left(B^{*}\right)}=$ $\mathcal{D}_{1(B)} \otimes \mathcal{D}_{2}$.

Proof: The point-set of $\mathcal{D}_{\left(B^{*}\right)}$ are: $B \cup \mathcal{P}_{2} \cup B \times \mathcal{P}_{2}$, which is also the point set of $\mathcal{D}_{1(B)} \otimes \mathcal{D}_{2}$. For blocks, $\left(B_{1}, B_{2}\right) \cap B^{*}=\left(B_{1} \cap B, B_{2}\right)$.

Corollary 4.17. Let $\mathcal{D}=\mathcal{D}_{1} \sigma \mathcal{A}_{2}$ have a unique good block and no good points. If $\mathcal{D}_{1}$ is tensor-indecomposable, so is $\mathcal{D}$.

Proof: Since $\mathcal{D}$ has no good points, $\sigma \neq \sigma_{0}$, so $\mathcal{D}$ is not trivially decomposable. Suppose $\mathcal{D}=\mathcal{D}_{3} \otimes \mathcal{D}_{4}$. By the dual of Lemma 4.8, we may assume the unique good block of $\mathcal{D}$ is induced by a good block $B$ of $\mathcal{D}_{3}$. Thus, the good block is a block of the form $B^{*}$, so by the Lemma above, $\mathcal{D}_{\left(B^{*}\right)}=\mathcal{D}_{3(B)} \otimes \mathcal{D}_{4}$. However, by (3.1) we have $\mathcal{D}_{\left(B^{*}\right)} \cong \mathcal{D}_{1}$, which is tensor-indecomposable by assumption. 


\section{Constructing rigid designs}

In this section we construct Hadamard designs with no non-trivial automorphisms. First, we mention a well-known fact regarding automorphisms of Hadamard designs.

Fact 5.1. Let $\mathcal{D}$ be a $2-(4 n-1,2 n-1, n-1)$ Hadamard design and $1 \neq g \in$ $\operatorname{Aut}(\mathcal{D})$. Then $g$ fixes at most $2 n$ points and at most $2 n$ blocks.

Proof: Choose a block $B$ such that $B^{g}=C \neq B$. Therefore $h: B \cap C^{c} \rightarrow B^{c} \cap C$, and $\left|B \cap C^{c}\right|=\left|B^{c} \cap C\right|=n$, so the support of $g$ contains at least $2 n$ points. Thus, $g$ fixes at most $4 n-1-2 n=2 n$ points. Dually, $g$ fixes at most $2 n$ blocks.

Theorem 5.2. If a Hadamard design of order $n>2$ exists, there exist at least $\frac{(16 n-2) !}{2^{10} n^{3}}$ pairwise non-isomorphic, tensor-indecomposable Hadamard designs of order $8 n$ with exactly one good block, no good points, and no non-trivial automorphisms.

Proof: By Theorem 3.8, we may assume $\mathcal{D}$ has no good blocks. Set $\mathcal{D}_{1}=\mathcal{D} \sigma\left(\mathcal{D}^{*}\right)^{+}$, where $\sigma$ is chosen as in Corollary 3.12 so there exists a point $q$ of $\mathcal{D}_{1}$ which is on no lines of size 3 . We list some properties of $\mathcal{D}_{1}$.

1.1. $\mathcal{D}_{1}$ has a unique good block, $B_{\infty}$. For, $\mathcal{D}$ has no good blocks, so Eq. (3.3) rules out any other good blocks.

1.2. $\mathcal{D}_{1}$ has no good points. For, a good point would be on a line of size 3 with $q$, which is ruled out.

1.3. Any automorphism inducing the identity on $B_{\infty}$ is the identity. For, any such automorphism would fix the parallel classes of $D_{1}^{B_{\infty}}=\left(\mathcal{D}^{*}\right)^{+}$, hence induce a translation of that affine design. However, $\mathcal{D}$ has no good blocks, so $\mathcal{D}^{*}$ has no good points. Therefore, Lemma 2.1 implies $\left(\mathcal{D}^{*}\right)^{+}$has no translations.

1.4. $\mathcal{D}_{1}$ is tensor-indecomposable. For, the point $q$ is on no lines of size 3 , hence use Corollary 4.5.

We now define $\mathcal{D}_{2}=\mathcal{D}_{1} \tau \mathcal{D}_{1}^{+}$for a carefully chosen $\tau$. Let $\hat{B}$ denote the unique good block of $\mathcal{D}_{1}$ (this notation is used so as not to confuse it with the new block $B_{\infty}$ of $\left.\mathcal{D}_{2}\right)$. Fix a point of $p \in \hat{B}$, so that $p$ is on $2 n-1$ blocks of $\mathcal{D}_{1(\hat{B})}=\mathcal{D}$. These blocks are of the form $B_{i} \cap \hat{B}$, where $B_{i} \neq \hat{B}$ is a block of $\mathcal{D}_{1}$ containing $p$. So, let $B_{1}, B_{2}, \ldots, B_{2 n-1}$ be $2 n-1$ blocks inducing those blocks of $\mathcal{D}_{1(\hat{B})}$ (note that we have 2 choices for each block). Let $C$ be an arbitrary block not containing $p$. Set $\tau=\sigma_{0} t$ where $\sigma_{0}$ is as in note 3.5 and $t$ is the following element of the symmetric group on the blocks of $\mathcal{D}_{1}$.

$$
t=\left(\hat{B}, B_{1}, B_{2}, \ldots, B_{2 n-2}, C\right) .
$$

Properties of $\mathcal{D}_{2}$ :

2.1. $B_{\infty}$ is the unique good block of $\mathcal{D}_{2}$. Recall that Eq. (3.3) shows that any other good block must arise as the union of a good block of $\mathcal{D}_{1}^{+}$and a good block of $\mathcal{D}_{1}$. Since $\hat{B} \in \operatorname{supp}(t)$, this does not happen, as although $\hat{B}$ is good, $\hat{B}^{t}=B_{1}$ is not. 
2.2. $\mathcal{D}_{2}$ has no good points on $B_{\infty}$. Otherwise, the elation given by Theorem 3.7 would induce a translation of $\mathcal{D}_{2}^{B_{\infty}}=\mathcal{D}_{1}^{+}$, which is impossible by Lemma 2.1 since $\mathcal{D}_{1}$ has no good points, by Property 1.2 of $\mathcal{D}_{1}$.

2.3. $\mathcal{D}_{2}$ has no good points off $B_{\infty}$. For, such a good point would imply that $\mathcal{D}_{2} \cong \mathcal{D}_{1} \sigma_{0} \mathcal{D}_{1}^{+}$by Theorem 3.6. Setting $G_{1}=\operatorname{Aut}\left(\mathcal{D}_{1}\right)$ in its permutation representation on the blocks of $\mathcal{D}_{1}$ and $\overline{G_{1}}=\operatorname{Aut}\left(\mathcal{D}_{1}^{+}\right)$in its representation on the parallel classes of blocks of $\mathcal{D}_{1}^{+}$, the assumed isomorphism and Fact 3.4 give $\tau \in \overline{G_{1}} \sigma_{0} G_{1}$.

On the other hand, $\overline{G_{1}}$ fixes $\overline{\hat{B}}$, since it is the unique good parallel class of $\mathcal{D}_{1}^{+}$, and $G_{1}$ fixes $\hat{B}$, since it is the unique good block of $\mathcal{D}_{1}$. Thus, any element of $\overline{G_{1}} \sigma_{0} G_{1}$ sends $\overline{\hat{B}}$ to $\hat{B}$. However, $\overline{\hat{B}}^{\tau}=B_{1}$, so no isomorphism is possible.

2.4. $\mathcal{D}_{2}$ is tensor-indecomposable by Corollary 4.17 , as $\mathcal{D}_{1}$ is indecomposable, by Property 1.4 .

2.5. $\mathcal{D}_{2}$ has a point moved by any non-trivial automorphism.

Proof: We'll show $p_{\infty}$ behaves as stated: if $g \in \operatorname{Aut}\left(\mathcal{D}_{2}\right)$ fixes $p_{\infty}$, we will show $g=1$.

By Corollary 3.10, $g$ induces a $g_{1} \in G_{1}$ and a $g_{2} \in \operatorname{Aut}\left(\mathcal{D}^{+}\right)$which induces a $\overline{g_{2}} \in \overline{G_{1}}$ on parallel classes ( $G_{1}$ and $\overline{G_{1}}$ as in Property 2.3 above), with $g_{1}={\overline{g_{2}}}^{\tau}$. Since $g$ fixes $p_{\infty}$, so does $g_{2}$. Thus, $g_{2}$ fixes (as a set) the blocks on $p_{\infty}$, and restricted to those blocks $g_{2}$ induces an automorphism $g_{2}^{*}$ of $\left(\mathcal{D}_{1}^{+}\right)_{p_{\infty}}=\mathcal{D}_{1}$. Since one member of each parallel class contains $p_{\infty}$, the action of $g_{2}^{*}$ on those blocks can be deduced from the action of $\overline{g_{2}}$ on parallel classes. In fact, since $\sigma_{0}$ associates to each parallel class of $\mathcal{D}_{1}^{+}$the member of the parallel class containing $p_{\infty}$, we see $g_{2}^{*}={\overline{g_{2}}}^{\sigma_{0}}$ in its representation on blocks of $\mathcal{D}_{1}$.

Since $g_{1}={\overline{g_{2}}}^{\tau}={\overline{g_{2}}}^{\sigma_{0} t}=\left(g_{2}^{*}\right)^{t}$, we have:

$$
g_{1}\left(g_{2}^{*}\right)^{-1}={\overline{g_{2}}}^{\tau}\left(g_{2}^{*}\right)^{-1}=t^{-1}{\overline{g_{2}}}^{\sigma_{0}} t\left(g_{2}^{*}\right)^{-1}=t^{-1} t^{\left(g_{2}^{*}\right)^{-1}} .
$$

A priori, the support of $g_{1}\left(g_{2}^{*}\right)^{-1}=t^{-1} t^{\left(g_{2}^{*}\right)^{-1}}$ is at most twice the size of the support of $t$. However, both of the automorphisms $\left(g_{2}^{*}\right)^{-1}$ and $g_{1}\left(g_{2}^{*}\right)^{-1}$ must fix $\hat{B}$. Thus,

$$
\hat{B}^{g_{1}\left(g_{2}^{*}\right)^{-1}}=\hat{B}^{t^{-1} g_{2}^{*} t^{-1}\left(g_{2}^{*}\right)^{-1}}=\hat{B}
$$

implies:

$$
\hat{B}^{t^{-1}}=C=\hat{B}^{g_{2}^{*} t^{-1}\left(g_{2}^{*}\right)^{-1}}=C^{\left(g_{2}^{*}\right)^{-1}}
$$

so $g_{2}^{*}$ fixes $C$. Therefore,

$$
\left|\operatorname{supp}\left(g_{1}\left(g_{2}^{*}\right)^{-1}\right)\right| \leq 2(|\operatorname{supp}(t)|-1)=4 n-2 .
$$

Thus, $g_{1}\left(g_{2}^{*}\right)^{-1}$ fixes at least $4 n+1$ blocks, implying $g_{1}\left(g_{2}^{*}\right)^{-1}=1$, by Fact 5.1 since $\mathcal{D}_{1}$ is of order $2 n$. So, $g_{1}=g_{2}^{*}$ and $g_{1}$ commutes with $t$. Since $g_{1}$ 
centralizes $t$ and fixes $\hat{B}, g_{1}$ must fix all elements of $\operatorname{supp}(t)$. Thus, $g_{1}$ induces an automorphism $g_{1}^{*}$ of $\mathcal{D}_{1(\hat{B})}$ fixing all but at most one block on $p$, so $g_{1}^{*}$ must fix all blocks on $p$ (since $\lambda<k-1$ for any Hadamard design). Additionally, $g_{1}^{*}$ fixes the block $C \cap \hat{B}$ of $\mathcal{D}_{1(\hat{B})}$ which means $g_{1}^{*}$ fixes at least $2 n$ blocks of $\mathcal{D}_{1(\hat{B})}$. Thus, by Fact $5.1, g_{1}^{*}$ is the identity, as $\mathcal{D}_{1(\hat{B})}=\mathcal{D}$ is of order $n$. Thus, $g_{1}$ induces the identity on $\mathcal{D}_{1(\hat{B})}$ and thus $g_{1}$ is the identity on $\mathcal{D}_{1}$ by Property 1.3 of $\mathcal{D}_{1}$. Since $g_{1}$ is the identity on blocks, $\overline{g_{2}}=g_{1}^{\gamma^{-1}}$ is the identity on parallel classes, meaning $g_{2}$ is the identity, or a translation. Since $g_{2}$ fixes $p_{\infty}$, it must be the identity (recall that translations are fixed point free). Thus, any $g \in \operatorname{Aut}\left(\mathcal{D}_{2}\right)$ which fixes the point $p_{\infty}$ must be the identity.

2.6. $\left|\operatorname{Aut}\left(\mathcal{D}_{2}\right)\right| \leq 8 n$ and $\left|\operatorname{Aut}\left(\mathcal{D}_{2}^{+}\right)\right| \leq 16 n(8 n)$. Since $p_{\infty}$ is fixed by no non-trivial automorphism, $\left|\operatorname{Aut}\left(\mathcal{D}_{2}\right)\right|$ is equal to the size of the orbit of $p_{\infty}$, which is at most $8 n$, since $p_{\infty}$ is off the unique good block of $\mathcal{D}_{2}$. Aut $\left(D_{2}^{+}\right)$is bounded similarly, as $\mathcal{D}_{2}^{+}$is formed by adding another point, whose orbit size is at most $16 n$.

Since $\mathcal{D}_{2}$ has a point not fixed by any non-trivial automorphism, $\mathcal{D}_{2}^{*}$ has a block with that property, call this block $\tilde{B}$. Also, $\mathcal{D}_{2}$ has a unique good block we'll call $C_{2}$ (again, so as not to confuse it with the new $B_{\infty}$ in the following construction) which induces the unique good parallel class $\overline{C_{2}}$ of $\mathcal{D}_{2}^{+}$. Set $\mathcal{D}_{3}=\mathcal{D}_{2}^{*} \gamma \mathcal{D}_{2}^{+}$where ${\overline{C_{2}}}^{\gamma}=\tilde{B}$, and $\gamma$ is otherwise arbitrary. Note that there are $(16 n-2)$ ! choices for $\gamma$, as $\mathcal{D}_{2}$ is of order $4 n$.

$\mathcal{D}_{3}$ has the following properties:

3.1. $\mathcal{D}_{3}$ has only one good block. For, since $\mathcal{D}_{2}$ has no good points by 2.2 and $2.3, \mathcal{D}_{2}^{*}$ has no good blocks, so again, Eq. (3.3) ensures that $B_{\infty}$ is the only good block of $\mathcal{D}_{3}$.

3.2. $\mathcal{D}_{3}$ has no good points on $B_{\infty}$. For, just as with the construction of $\mathcal{D}_{2}$, such a good point would induce an elation by Theorem 3.7 , which would induce a translation of $\mathcal{D}_{3}^{B_{\infty}}=\mathcal{D}_{2}^{+}$. This is impossible by Lemma 2.1 , since $\mathcal{D}_{2}$ has no good points, again by 2.2 and 2.3 .

3.3. $\mathcal{D}_{3}$ has no good points off $B_{\infty}$. Otherwise by Theorem 3.7, we would have $\mathcal{D}_{3} \cong \mathcal{D}_{2}^{*} \sigma_{0} \mathcal{D}_{2}^{*+}$, which would imply $\left(\mathcal{D}_{3}\right)_{B_{\infty}}=\mathcal{D}_{2}^{+} \cong \mathcal{D}_{2}^{*+}$. But, $\mathcal{D}_{2}^{+}$has a good parallel class, $\overline{C_{2}}$. However, $\mathcal{D}_{2}$ has no good points, by Properties 2.2 and 2.3, so $\mathcal{D}_{2}^{*}$ has no good blocks, so $\mathcal{D}_{2}^{*+}$ has no good parallel classes. Thus, $\mathcal{D}_{2}^{*+} ¥ \mathcal{D}_{2}^{+}$.

3.4. $\mathcal{D}_{3}$ is tensor-indecomposable by Corollary 4.17 , as $\mathcal{D}_{2}$ is, by 2.4 .

3.5. $\operatorname{Aut}\left(\mathcal{D}_{3}\right)=1$. For, any $g \in \operatorname{Aut}\left(\mathcal{D}_{3}\right)$ induces a $g_{2} \in \operatorname{Aut}\left(\mathcal{D}_{2}^{+}\right)$, acting as $\overline{g_{2}}$ on parallel classes, which must fix $\overline{C_{2}}$ (as $\overline{C_{2}}$ is the unique good parallel class of $\left.\mathcal{D}_{2}^{+}\right)$, and a $g_{1} \in \operatorname{Aut}\left(\mathcal{D}_{2}^{*}\right)$. By Lemma 3.9: $g_{1}=\bar{g}_{2}^{\gamma}$, and since $\bar{C}^{\gamma}=\tilde{B}, g_{1}$ must fix $\tilde{B}$, implying that $g_{1}$ is the identity (because $\tilde{B}$ was chosen to be the block moved by any non-trivial automorphism). Thus, $\overline{g_{2}}$ is the identity on parallel classes of blocks. Since $\mathcal{D}_{2}^{+}$does not admit translations (again by Lemma 2.1 and the fact $\mathcal{D}_{2}$ has no good points, by 2.2 and 2.3$), g_{2}$ must be the identity. Thus, $g$ fixes all blocks of $\mathcal{D}_{3}$, so must be the identity.

3.6. There are at least $\frac{(16 n-2) !}{2^{10} n^{3}}$ non-isomorphic choices for $\mathcal{D}_{3}$. Clearly there are $(16 n-2)$ ! choices for $\gamma$. By Fact 3.4, a given $\mathcal{D}_{2}^{*} \gamma \mathcal{D}_{2}^{+}$can only be isomorphic to $\left|\operatorname{Aut}\left(\mathcal{D}_{2}^{*}\right) \gamma \operatorname{Aut}\left(\mathcal{D}_{2}^{+}\right)\right| \leq 2{ }^{10} n^{3}$ other such designs, by the bounds in Property 2.6. 


\section{GMW designs}

GMW designs are symmetric designs arising from certain difference sets, and have the same parameters as projective spaces. For a definition and basic properties, see [2, Section VI, 17]. Additionally, [8, Theorem 1] gives the full automorphism group of such designs, as well as determining when they are isomorphic. This is also proven in [3, Theorem 1.2], without the use of the classification of finite simple groups. We will not need the full generality of the theorems in those papers, but use the following:

Fact 6.1. For any integer $N>1$, there exists a Hadamard GMW design $\mathcal{D}$ with parameters $2-\left(2^{3 N}-1,2^{3 N-1}-1,2^{3 N-2}-1\right)$, such that $\operatorname{Aut}(\mathcal{D}) \cong \Gamma \mathrm{L}(N, 8)$ in its natural action on non-zero vectors of $\mathbb{F}_{8}^{N}$.

The field size 8 here is entirely arbitrary, any power of 2 greater than 4 will do. Note that while the designs in question have the same parameters as a projective space of dimension $3 N-1$ over $\mathbb{F}_{2}$, they are not isomorphic to projective spaces, as their automorphism groups are too small. Other differences are also apparent.

Observation 6.2. The designs of Fact 6.1 have no good points or blocks, and are tensor-indecomposable.

Proof: Since the automorphism group is point/block transitive, one good point/block would imply that all points/blocks are good, contradicting the Dembowski-Wagner Theorem [2, Theorem XII.2.10], as these designs are not projective spaces. Also, the transitive automorphism group ensures that these designs are tensor-indecomposable by Lemma 4.10 .

Lemma 6.3. Let $\mathcal{D}=(\mathcal{P}, \mathcal{B})$ be a GMW design as given in Fact 6.1. Then Aut $\left(\mathcal{D}^{+}\right)=$ $\operatorname{Aut}(\mathcal{D})$.

Proof: Set $G=\operatorname{Aut}\left(\mathcal{D}^{+}\right)$. We need to show that $G$ fixes the point $p_{\infty}$. Assume that $p_{\infty}$ is moved. Then $G$ is 2 -transitive on points, as the subgroup fixing $p_{\infty}$ is already known to be transitive on the remaining points.

Claim: $G \cong \operatorname{A} \Gamma \mathrm{L}(N, 8)$. By 6.1 , we have $G_{p_{\infty}}=\operatorname{Aut}(\mathcal{D}) \cong \Gamma \mathrm{L}(N, 8)$. Consider the subgroup $T<G_{p_{\infty}}$ consisting of transvections fixing a common hyperplane (see [17, Ch. 4] for relevant definitions). $|T|=8^{N-1}, T$ fixes $8^{N-1}$ points (including $p_{\infty}$ ), and any other such subgroup of $G_{p_{\infty}} \cong \Gamma \mathrm{L}(N, 8)$ is conjugate to $T$ in $G_{p_{\infty}}$. Set $H=\operatorname{Fix}(T) \subset \mathcal{P} \cup\left\{p_{\infty}\right\}$ and consider the incidence structure $\mathcal{D}^{\prime}=\left(\mathcal{P} \cup\left\{p_{\infty}\right\}, H^{G}\right)$. Since $G$ is 2-transitive, this is a design. Some properties of $\mathcal{D}^{\prime}$ :

1. If $g \in G$ and $p_{\infty} \in H^{g}$, then $H^{g} \in H^{G_{p \infty}}$ as $H^{g}=\operatorname{Fix}\left(T^{g}\right)$, so $T$ and $T^{g}$ are $G_{p_{\infty}}$ conjugate.

2. Thus, the number of blocks of $\mathcal{D}^{\prime}$ on $p_{\infty}$ is the number of conjugates of $T$ in $G_{p_{\infty}}$, which in turn is the number of hyperplanes of a vector space of dimension $N$ over $\mathbb{F}_{8}$, which is $\frac{8^{N}-1}{7}$. Thus, $\mathcal{D}^{\prime}$ has parameters $v=8^{N}, k=|H|=8^{N-1}$, and 
$r=\frac{8^{N}-1}{7}$. Therefore, the parameters of $\mathcal{D}^{\prime}$ are the same as $\mathcal{A G}(N, 8)$. However, we do not yet know that the $\mathcal{D}^{\prime}$ is, in fact, classical.

3. Let $H_{1} \neq H_{2} \in H^{G}$ with $H_{1} \cap H_{2} \neq \phi$. By transitivity of $\operatorname{Aut}\left(\mathcal{D}^{\prime}\right)$, we may assume $p_{\infty} \in H_{1} \cap H_{2}$. The remaining points of $H_{1} \cap H_{2}$ are those points fixed by 2 distinct conjugates of $T$ in $G_{p_{\infty}}$. But these are just the non-zero points contained in the intersection of 2 hyperplanes of $\mathbb{F}_{8}^{N}$. Thus, $\left|H_{1} \cap H_{2}\right|=8^{N-2}$. Furthermore, there are exactly 9 conjugates of $T$ pointwise fixing $H_{1} \cap H_{2}$, so there are exactly 9 blocks containing $H_{1} \cap H_{2}$. Since these 9 blocks cannot pairwise intersect outside of $H_{1} \cap H_{2}$, they partition $\mathcal{P} \cup\left\{p_{\infty}\right\}-\left(H_{1} \cap H_{2}\right)$.

By [5, Theorem 1], the above 3 properties imply $\mathcal{D}^{\prime} \cong \mathcal{A G}(N, 8)$, proving the claim that $G \cong A \Gamma \mathrm{L}(N, 8)$. Therefore, $G \cong G_{p_{\infty}} \ltimes V$, where $V=\mathbb{F}_{8}^{N}$.

Finally, we return to the original design $\mathcal{D}^{+}$by considering the (point-transitive, hence point-regular) action of $V=\mathbb{F}_{8}^{N}$ on the blocks of $\mathcal{D}^{+}$. Since there are $2^{3 N-1}-1$ parallel classes in $\mathcal{D}^{+}, V$ must fix at least one: $\left\{B, B^{c}\right\}$. Then, $V$ has a subgroup $W$ of index 2 fixing $B$, hence acting regularly on the points of $B$. Thus, $B$ can be identified with $W$, a hyperplane of $\mathcal{A G}(V)$, so by transitivity, all blocks can be identified with hyperplanes. Therefore, $\mathcal{D}^{+} \cong \mathcal{A G}(3 n, 2)$, which in turn implies that $\mathcal{D} \cong \mathcal{P G}(3 N-$ $1,2)$, and $\operatorname{Aut}(\mathcal{D}) \cong G L(3 N, 2)$ contradicting Fact 6.1.

Note that this proof can also be done by simply citing [16, Theorem 1.1], which classifies all affine designs admitting a 2-transitive action on points. However, the above proof is included for completeness, and does not require the classification of finite simple groups.

\section{Isolating given finite groups}

In light of Theorem 3.10, we now show that any group is isomorphic to the intersection of two conjugate copies of $\Gamma \mathrm{L}(N, 8)$ within the symmetric group $\mathbf{S}_{8^{N}-1}$ for suitably chosen $N$. The lemma below is adapted from [8]. A similar argument can be found in [7, Lemma 10.3].

Lemma 7.1. Let $G$ be a finite group, with $N>4|G|+2$. There exists $\sigma \in \mathbf{S}_{8^{N}-1}$ such that $G \cong \Gamma \mathrm{L}(N, 8) \cap \Gamma \mathrm{L}(N, 8)^{\sigma}$.

Proof: Throughout, we will be considering the action of the groups $\mathbf{S}_{8^{N}-1}, G$ and $\Gamma \mathrm{L}(N, 8)$ on non-zero vectors of $\mathbb{F}_{8}^{N}$, so notation involving span and direct sum will always be assumed to have the zero vector removed. Let $K=\mathbb{F}_{8^{2}}$ and set

$$
\mathbb{F}_{8}^{N}=\left(\oplus_{g} K x_{g}\right) \oplus K u \oplus\langle Y\rangle,
$$

where $Y$ is some set of more than $2|G|$ linearly independent vectors. $G$ acts on $\mathbb{F}_{8}^{N}$ via $\left(K x_{g}\right)^{h}=K x_{g h}$ while pointwise fixing $K u$ and $\langle Y\rangle$. Fix $y_{0} \in Y_{0} \subseteq Y$ where $\left|Y_{0}\right|=$ $2|G|$.

Now, set $\pi$ to be the product of 2 disjoint cycles $\pi_{1}$ and $\pi_{2}$, both pointwise fixing $K x_{1}$ and $K u$, with $\pi_{1}$ a 6-cycle permuting all but 1 non-zero vector of a 1 -space of 會Springer 
$K x_{1} \oplus K u$, and $\pi_{2}$ a $\left(8^{4}-2\left(8^{2}\right)-5\right)$-cycle acting on the remaining non-zero vectors of $K x_{1} \oplus K u$. Set $\pi^{\prime}$ to be the product of 2 disjoint cycles $\pi_{1}^{\prime}$ and $\pi_{2}^{\prime}$, both pointwise fixing $K x_{1}$ and $K y_{0}$, with $\pi_{1}^{\prime}$ a 6-cycle permuting all but 1 non-zero vector of a 1 space of $K x_{1} \oplus K y_{0}$ and $\pi_{2}^{\prime}$ a $\left(8^{4}-2\left(8^{2}\right)-6\right)$-cycle on $K x_{1} \oplus K y_{0}$, whose support contains the remaining non-zero vector of the 1 -space spanned by the support of $\pi_{1}^{\prime}$. Set $\pi_{*}$ to be the product of 2 disjoint cycles $\pi_{1 *}$ and $\pi_{2 *}$, both pointwise fixing $K u$ and $\langle Y\rangle$, where $\pi_{1 *}$ is a 6-cycle on all but 1 non-zero vector of a 1 -space, and $\pi_{2 *}$ a $\left(8^{2+|Y|}-8^{|Y|}-8^{2}-5\right)$-cycle on the remaining non-zero vectors of $K u \oplus\langle Y\rangle$. Whenever $1 \neq g \in G$ let $\pi_{g}$ denote the product of 2 disjoint cycles, both pointwise fixing $K x_{1} \oplus\left\langle x_{g}\right\rangle$ and $K x_{1} \oplus\left\langle Y_{0}\right\rangle, \pi_{1 g}$ is a 6-cycle permuting all but 1 non-zero vector of a 1-space, and $\pi_{2 g}$ a cycle of length greater than $8^{2+\left|Y_{0}\right|}$ whose support spans exactly $K x_{1} \oplus\left\langle x_{g}\right\rangle \oplus\left\langle Y_{0}\right\rangle$. Furthermore, choose $\pi_{2 g}$ in such a way that the length of the cycle differs for distinct $g$. This is possible, as $|G|<8^{3+\left|Y_{0}\right|}-\left(8^{3}+8^{\left|Y_{0}\right|}+8^{2+\left|Y_{0}\right|}+6\right)$. For all $h \in G$ define $\sigma$ to be

- $\pi^{h}$ on $\left(K x_{1} \oplus K u\right)^{h}=K x_{h} \oplus K u$,

- $\pi^{\prime h}$ on $\left(K x_{1} \oplus K y_{0}\right)^{h}=K x_{h} \oplus K y_{0}$,

- $\pi_{g}^{h}$ on $\left(K x_{1} \oplus\left\langle x_{g}\right\rangle \oplus\left\langle Y_{0}\right\rangle\right)^{h}=K x_{h} \oplus\left\langle x_{g h}\right\rangle \oplus\left\langle Y_{0}\right\rangle$ whenever $g \neq 1$, and

- $\pi_{*}$ on $K u \oplus\langle Y\rangle$.

Then: $G \leq \Gamma \mathrm{L}(N, 8) \cap \Gamma \mathrm{L}(N, 8)^{\sigma}$, as all elements of $G$ commute with $\sigma$; we are going to prove equality here.

Now, suppose we have $\alpha, \beta \in \Gamma \mathrm{L}(N, 8)$ such that $\alpha=\beta^{\sigma}$. Then we have $\alpha^{-1} \beta=$ $\sigma^{-1} \sigma^{\beta} \in \Gamma L(N, 8)$. But the support of $\sigma^{-1} \sigma^{\beta}$ has size at most:

$$
2|\operatorname{supp}(\sigma)| \leq 2\left(8^{4} 2|G|+8^{3+\left|Y_{0}\right|}|G|(|G|-1)+8^{2+|Y|}\right)<8^{N}-8^{N-1}
$$

and therefore $\alpha^{-1} \beta$ is the identity, as no non-trivial element of $\Gamma \mathrm{L}(N, 8)$ can fix more than $8^{N-1}$ non-zero vectors of $\mathbb{F}_{8}^{N}$. Thus, $\sigma$ centralizes $\alpha$. Then $\alpha$ permutes the cycles of $\sigma$. Since $8^{2+|Y|}-8^{|Y|}>8^{3+\left|Y_{0}\right|}-8^{2+\left|Y_{0}\right|}, \pi_{2 *}$ is a longer cycle than any of the $\pi_{2 g}$, and hence is the longest cycle of $\sigma$. Thus, $\alpha$ must stabilize the support of $\pi_{2 *}$. Also, $\alpha$ permutes the 6 -cycles of $\sigma$, hence must permute the 1 -spaces they determine. Thus, $\alpha$ must fix the single vector in the intersection of those 1-spaces and the support of $\pi_{2 *}$. Since $\alpha$ fixes this vector and commutes with $\pi_{2 *}$, it must fix all vectors in the support of $\pi_{2 *}$. This set contains a basis for $K u \oplus\langle Y\rangle$, so $\alpha$ must be the identity on this subspace. In particular, $\alpha$ is linear.

If $\left(K x_{1} \oplus K u\right)^{\alpha}=K x_{h} \oplus K u$ then we may replace $\alpha$ with $\alpha h^{-1}$ and assume $K x_{1} \oplus K u$ is left invariant by $\alpha$. This means that $\alpha$ commutes with $\pi$, hence must stabilize the support of $\pi_{2}$. Again, this support intersects the 1-spaces determined by all the 6-cycles of $\sigma$ in a single vector, implying that $\alpha$ pointwise fixes the support of $\pi_{2}$. Again, this set contains a basis of $K x_{1} \oplus K u$, so $\alpha$ induces the identity on $K x_{1} \oplus K u$. Note that if $|G|=1$ we are finished, as in that case $\mathbb{F}_{8}^{N}=K x_{1} \oplus K u \oplus\langle Y\rangle$, so we have shown that $\alpha$ is the identity. Therefore, we may assume $|G|>1$.

$\alpha$ must permute the cycles of length $8^{4}-2\left(8^{2}\right)-5$, as well as the cycles of length $8^{4}-2\left(8^{2}\right)-6$. Thus, for all $h \in G$, there exists $h^{*}, \bar{h} \in G$ satisfying $\left(K x_{h} \oplus K u\right)^{\alpha}=K x_{\bar{h}} \oplus K u$ and $\left(K x_{h} \oplus K y_{0}\right)^{\alpha}=K x_{h^{*}} \oplus K y_{0}$. Then $\left(K x_{h}\right)^{\alpha} \subseteq$ 
$\left(K x_{\bar{h}} \oplus K u\right) \cap\left(K x_{h^{*}} \oplus K y_{0}\right)$ implies that $\left(K x_{h}\right)^{\alpha}=K x_{h^{*}}=K x_{\bar{h}}$, since $K u$ and $K y_{0}$ are fixed by $\alpha$. Thus, the "basic" subspaces $K x_{h}$ are permuted by $\alpha$.

Let $g \neq 1$. Then the cycles $\pi_{2 g}$ are of distinct length for different $g . \alpha$ must permute these cycles, so for each $h$ there is some $h^{\prime}$ such that

$$
\left(K x_{h} \oplus\left\langle x_{g h}\right\rangle \oplus\left\langle Y_{0}\right\rangle\right)^{\alpha}=K x_{h^{\prime}} \oplus\left\langle x_{g h^{\prime}}\right\rangle \oplus\left\langle Y_{0}\right\rangle
$$

Then $K x_{h^{*}} \oplus\left\langle x_{g h}\right\rangle^{\alpha} \subset K x_{h^{\prime}} \oplus\left\langle x_{g h^{\prime}}\right\rangle \oplus\left\langle Y_{0}\right\rangle$ so that $h^{\prime}=h^{*}$, since $K x_{h^{\prime}}$ is the only "basic" subspace of $K x_{h^{\prime}} \oplus\left\langle x_{g h^{\prime}}\right\rangle \oplus\left\langle Y_{0}\right\rangle$. This in turn implies that $\left\langle x_{g h}\right\rangle^{\alpha}=\left\langle x_{g h^{\prime}}\right\rangle$. Since $\left\langle x_{g h}\right\rangle^{\alpha} \subset\left(K x_{g h}\right)^{\alpha}=K x_{(g h)^{*}}$, it follows that $(g h)^{*}=g h^{\prime}=g h^{*}$ for all $g \neq 1$ and $h$. Since $1^{*}$ is already known to be 1 , setting $h=1$, we see $g^{*}=g$, so that $\alpha$ stabilizes each $K x_{g}$. Thus, $\left(K x_{g} \oplus K u\right)^{\alpha}=K x_{g} \oplus K u$, since $\alpha=1$ on $K u$. As before, this means that $\alpha$ must centralize $\pi_{2}^{h}$, and fix the vector it has in common with the 1-spaces determined by the 6-cycles, so $\alpha$ must pointwise fix the support of $\pi_{2}^{h}$. Again, this support contains a basis for $K x_{h} \oplus K u$, and therefore $\alpha=1$ on this subspace. Thus, $\alpha$ is the identity, and we have shown $G=\Gamma \operatorname{L}(N, 8) \cap \Gamma \operatorname{L}(N, 8)^{\sigma}$.

Corollary 7.2. For any finite group $G$ and $N>4|G|+2$, there exists a tensorindecomposable Hadamard design on $v=2^{3 N+1}-1$ points with automorphism group isomorphic to $G$, a unique good block and no good points.

Proof: Let $\mathcal{D}$ be a GMW design on $2^{3 N}-1$ points with $\operatorname{Aut}(\mathcal{D}) \cong \Gamma \mathrm{L}(N, 8)$, as in Fact 6.1. Since $\mathcal{D}$ is an abelian difference set design, it is self-dual. Thus, the permutation $\sigma$ defined in Lemma 7.1, which acts on the points of $\mathcal{D}$, can be dualized to a permutation $\sigma^{*}$ which acts on blocks of $\mathcal{D}$. Form $\mathcal{D}_{G}=\mathcal{D} \hat{\sigma} \mathcal{D}^{+}$, where $\hat{\sigma}=\sigma_{0} \sigma^{*}$. $\mathcal{D}_{G}$ has $2^{3 N+1}-1$ points. Since $\mathcal{D}$ has no good blocks by Observation $6.2, B_{\infty}$ is the unique good block of $\mathcal{D} \sigma \mathcal{D}^{+}$by Eq. (3.3). Thus, Corollary 3.10 gives $\operatorname{Aut}\left(\mathcal{D} \sigma \mathcal{D}^{+}\right) \cong G$ as $\operatorname{Aut}(\mathcal{D})=\operatorname{Aut}\left(\mathcal{D}^{+}\right)$by Lemma 6.3. A good point off $B_{\infty}$ would yield $\mathcal{D}_{G} \cong \mathcal{D} \sigma_{0} \mathcal{D}^{+}$ by Fact 3.6. However, $\Gamma \mathrm{L}(N, 8) \leq \operatorname{Aut}\left(\mathcal{D} \sigma_{0} \mathcal{D}^{+}\right)$, stabilizing $B_{\infty}$, by Corollary 3.10 and $\Gamma \mathrm{L}(N, 8)$ is much larger than $G$. A good point on $B_{\infty}$ would induce an elation by Theorem 3.7 which in turn would induce a translation of $\mathcal{D}^{+}$, contradicting Fact 2.1 and Observation 6.2. Since $\mathcal{D}$ is tensor-indecomposable by Observation 6.2, so is $\mathcal{D}_{G}$ by Corollary 4.17 .

Proof of Theorem 1.1: Given a Hadamard design $\mathcal{D}$ of order $n$, form $\mathcal{D}_{3}$ of order $8 n$ with $\operatorname{Aut}\left(\mathcal{D}_{3}\right)=1$ as in Theorem 5.2. Given $G$, form $\mathcal{D}_{G}$ of order $2^{3 N-1}$ with $\operatorname{Aut}\left(\mathcal{D}_{G}\right) \cong G$ as in Corollary 7.2. Since neither $\mathcal{D}_{3}$ nor $\mathcal{D}_{G}$ has good points, we have $\operatorname{Aut}\left(\mathcal{D}_{G} \otimes \mathcal{D}_{3}\right) \cong G$ by Corollary $4.15 . \mathcal{D}_{G} \otimes \mathcal{D}_{3}$ is a Hadamard design of order $2^{3 N+4} n$. There are $\frac{(16 n-2) !}{2^{10} n^{3}}$ non-isomorphic choices for $\mathcal{D}_{3}$, inducing non-isomorphic designs $\mathcal{D}_{3} \otimes \mathcal{D}_{G}$ by the unique factorization of Corollary 4.14 .

Acknowledgements The author wishes to thank Bill Kantor for motivating this work and providing many helpful suggestions. 


\section{References}

1. L. Babai, "On the abstract group of automorphisms," in Combinatorics, Proc. Eighth British Comb. Conf. H.N.V. Temperley (Ed.), Cambridge U. Press, Cambridge, 1981 pp. 1-40.

2. T. Beth, D. Jungnickel, and H. Lenz, Design Theory, Cambridge University Press, Cambridge, 1999.

3. U. Dempwolff and W.M. Kantor, Distorting symmetric designs. in preparation

4. D. Jungnickel, "The number of designs with classical parameters grows exponentially," Geometriae Dedicata 16 (1984), 167-178.

5. W.M. Kantor, "Characterizations of finite projective and affine spaces," Can. J. Math. 21 (1969), 64-75.

6. W.M. Kantor, “Automorphism Groups of Hadamard Matrices,” J. Comb. Theory 6 (1969), 279-281.

7. W.M. Kantor, "Automorphisms and isomorphisms of symmetric and affine designs," J. Alg. Comb. 3 (1994), 301-338.

8. W.M. Kantor, "Note on GMW designs," Europ. J. Comb. (22) (2001), 63-69.

9. W.M. Kantor, unpublished manuscript.

10. M.E. Kimberley, "On the construction of certain Hadamard designs," Math Z. 119 (1971), 41-59.

11. C. Lam, S. Lam, and V.D. Tonchev, "Bounds on the number of Hadamard designs of even order," $J$. Comb. Designs 9 (2001), 363-378.

12. E. Merchant, "Exponentially many Hadamard designs," Des. Codes. and Cryptogr. 38(2) (2006), 297308.

13. C.W. Norman, "Hadamard designs with no non-trivial automorphisms," Geom. Ded. 2 (1976), 201-204.

14. C.W. Norman, "Non-isomorphic Hadamard designs," J. Combin. Theory (A) 21 (1976), 336-344.

15. R.E.A.C. Paley, “On Orthogonal Matrices,” J. Math. Phys. 12 (1933), 311-320.

16. O. Pfaff, "The classification of doubly transitive affine designs," Des., Codes, Cryptogr. 1 (1991), 207-217.

17. D.E. Taylor, The Geometry of the Classical Groups, Heldermann Verlag, Berlin, 1992.

18. J.A. Todd, “A combinatorial problem,” J. Math. Phys. 12 (1933), 321-333. 